\title{
A Case Study on the Advantages of 3D Walkthroughs over Photo Stitching Techniques
}

\author{
Kieran Latham ${ }^{(1)}$, William Hurst ${ }^{(1)}$, Nathan Shone ${ }^{(1)}$, Abdennour El Rhalibi ${ }^{(1)}$, Zhigeng Pan ${ }^{(2)}$ \\ (1) Department of Computer Science \\ Liverpool John Moores University \\ Byrom Street \\ Liverpool, L3 3AF, UK \\ (2) Digital Media and Interaction(DMI) Research Centre, \\ Hangzhou Normal University, \\ China \\ zgpan@cad.zju.edu.cn
}

K.Latham@2013.ljmu.ac.uk, \{W.Hurst, N.Shone,

A.Elrhalibi\}@ljmu.ac.uk

\begin{abstract}
Virtual tours and interactive walkthroughs enable a more in-depth platform for communicating information. Many current techniques employ the use of Photo Stitching to accomplish this. However, over the last decade advancements in computing power and the accessibility of game engines, meant that developing rich $3 \mathrm{D}$ content for virtual tours is more possible than ever before. As such, the purpose of this paper is to present a study into the advantages of developing an interactive 3D virtual tour of student facilities, using the Unreal Development 4 Game Engine, for educational establishments. The project aims to demonstrate a comparison between the use of Photo Stitching and 3D Modelled interactive walkthrough for developing rich visual environments. The research reveals that the approach in this paper can improve educational facilities prominence within universities, and contains many advantages over Photo Stitching techniques.
\end{abstract}

Index Terms - Virtual Tour, 3D Modelling, Animation, Education

\section{INTRODUCTION}

The use of visualisation and virtual reality can change the way in which we see and interpret data in the world. The methods, in which data is interacted with, can be essential for comprehending information. Using 3D technologies simplifies this process. It assists with understanding environments around us and is used as a distribution metric for sharing information readily with others. The current uses of 3D technology for data processing are diverse; ranging from visualising big data sets to performing computer network modelling [1]. Virtual tours are proven to have the ability to be applied to a number of applications outside of video games (the industry that has taken advantage of this technology for many years) such as tourism, education and military. The applications are varied. However, one commonality remains. The technology can be used for the knowledge sharing process. A complex dataset, or a real-world environment, can be understood and shared with others, once visualisation techniques are applied $[1,2]$.

The growing use of 3D technology to create walkthroughs and digitalise museums is an example of this. Information access and knowledge transfer are more widely accessible to anyone with an Internet connection. Existing projects, such as
CyArk, are an illustration of this [3]. They endeavour to preserve heritage data for future generations by developing an online 3D library of world cultural heritage sites. Other similar techniques are used by museums to share information with the wider online world. Projects, such as ARTtube, which profile European museums, digitalise collections using Photo Stitching to provide the global scientific community with access to data gathered [4].

However, current digital 'walkthroughs' extend beyond information sharing. In recent years, various museums exploit the use of information and communication technologies to enhance visitor experiences. With the emergence of innovative virtual reality devices, such as the Oculus Rift [5] and Microsoft HoloLens [6], it could be conceived that the uses of $3 \mathrm{D}$ immersive technology are receiving.

It is this feature that this paper transfers over to the creation of a tool for the visualisation of education establishment facilities. The techniques presented take advantage of the latest advances in software and hardware; particularly the accessibility of game engine and 3D modelling software. In this paper, the focus is on the development of a serious game, which can be used to educate and train individuals about 3D environments. The aim is to create a knowledge sharing tool, which digitalises university facilities and makes them more accessible to the wider audience.

Virtual reality technology combines the interaction between digital data with the users' view of the real world. The user is completely immersed in an artificial environment. As such, the research presented in this paper consists of four parts. Firstly, a literature review into a related research on serious games, gaming in education and the use of virtual tours is presented in Section 2. Secondly a case study into current related 3D walkthroughs is highlighted in Section 3. Thirdly, an approach for the production of $2 \mathrm{D}$ and $3 \mathrm{D}$ assets is put forward in Section 4. Finally, the development and evaluation of the interactive virtual tour, within a high level game engine, which allows the user to explore and interact with the environment, is detailed in Section 5. 


\section{BACKGROUND}

With the rising enthusiasm for the use of virtual reality technologies in academia, there has been an increase in research and case studies. In this section, related research projects are discussed and used as guidance for the creation of the tool presented in this paper.

\section{A. Related Projects}

The improved graphical capabilities of modern devices have significantly increased the popularity of 3D and virtual tours. Such solutions often offer significant benefits such as ease-of-use, wider dissemination potential and greater accessibility. Naturally, as a relatively infantile area, there are many emerging and ongoing research projects. Hence, in this section, we will examine some of the most current and pertinent research in this area.

Bonacini [7] presents a 360-degree tour of the Paolo Orsi Syracuse Archaeological Museum, which is based upon Google's Indoor Street view. It outlines the improved quality of Google's techniques for indoor tours, which offer significant improvements to the quality of the virtual tour by preventing collimation and image blurring. Similarly, Fineschi et al. [8] propose a 3D tour of the Santa Maria Della Scala Museum using the Oculus Rift virtual reality headset. This solution involved the 360-degree capture of images from the museum environment, which were combined into panoramic views and a $3 \mathrm{D}$ visitor path throughout the museum was overlaid onto this. Gonzalez-Delgado et al. [9] also propose a virtual 3D tour, focusing on paleontological heritage. Using geo-informatic tools, they created virtual tours of three georeferenced sites supported by multi-thematic geospatial information. They used various data sources such as digital terrain data and orthophotos data to create different thematic layers of satellite images of the area.

A project aimed at University tours is that proposed by Cosido et al. [10] whereby they created a prototype virtual geo-referenced tour with high precision positioning and geometry of objects and buildings. They utilize photogrammetry (obtaining measurements from images) and differential GPS data to obtain highly accurate threedimensional measurements and coordinates. Their motivation behind the work is to allow access to artefacts held in nonpublic locations and build a digitized archive so that in the eventuality of a building being damaged, it can be accurately reconstructed.

The work by Noordin et al. [11] bears some resemblance to the direction of the work outlined in this paper. They propose a 3D walkthrough virtual tourism application that can combine both 3D modelling and marker based augmented reality to overlay virtual $3 \mathrm{D}$ elements within the real environment.

There are also other elements of ongoing supplementary research, such as that by Zhang et al. [12], which is looking at using big data analytics to construct virtual tour schemas using image clustering of popular tour photos. There is also ongoing research examining the different ways in which to provide improved user experiences, for example, the work by Alletto et al. investigates the use of wearable cameras to provide a first person perspective [13]. Additionally, studies like those conducted by Chen and Mo [14] demonstrate the wide-ranging use of 3D tour solutions. In their study, they evaluate the use of a 3D tour as a training tool for new tour guides, with promising results.

\section{B. Digital Photo Stitching}

Most of the existing virtual tour solutions found during our literature review, involve the photographing of an environment to produce still 2D images, which are then assembled to form a pseudo-3D virtualised environment. This means that every part of the environment needs to be photographed, which is a timeconsuming task. Also, it requires high levels of accuracy in the photographs taken (e.g. positioning and height) to ensure smooth transitioning between images, without blurring or distortion. Although 2D images can provide a perfectly adequate virtual tour, they have a number of inherent limitations. Firstly, they lack a sense of realism, in that you cannot sense perspective, depth, texture and often scale, which can detract from the user experience and immersion into the environment. Secondly, the level of interactivity available in such approaches is often limited (such as clickable hotspots and modal boxes); hence, there is a need to provide extended interactive capabilities (e.g. ability to rotate artefacts through 360 degrees). Finally, exhibits and tourist attractions are often revised or restructured to ensure a continually engaging experience. However, this can cause problems when it comes to updating the virtual tours, as the whole environment will need to be re- photographed and re-processed.

\section{Digital Content Creation and Tools}

There are many types of digital content creation tools which provide different methods of creating digital content and application. The tools can be divided into $2 \mathrm{D}, 3 \mathrm{D}$, game engines and multimedia development platform, and some of the common digital creation content tools, in particular 3D tools, Flash, Unity 3D and UE4 are introduced below.

3D Tools - Digital content creation tools for 3D include Autodesk 3D Studio Max, Cinema 4D and Autodesk Maya for 3D modelling, animation and rendering. The tools are used to create $3 \mathrm{D}$ meshes as well as animations in $3 \mathrm{D}$ space. They are also used to render graphics and animations. Autodesk Mudbox, ZBrush and the free tool Blender are common tools for sculpting 3D meshes as well as texture painting. There are specialist tools for texture creation and texture.

Flash - Flash is a powerful tool that enables the creation and animation of videos, games, websites and applications that can combine all of these together. Multimedia is at the heart of Flash meaning it is encouraged that many different types of media are combined into one application. The multimedia application can then be enhanced with the use of ActionScript; coding to make objects animate or convert 
images into a game. Collision detection, camera control, navigation handlers, interactive buttons, material loaders and geometry loaders are just some of the features that will be utilized in the Virtual Tour when loaded into Flash.

Away 3D / Flare3D - These APIs are a Real-Time 3D engine for Flash. They support animation, camera, lighting, Level of Details (LOD) and a range of other features seen in conventional 3D graphic engine. A graphic engine is a software framework which consists of methods that enable real-time $3 \mathrm{D}$ applications to be rapidly created. Using a 3D graphic engine means you have to use the methods in the library and feeding them the data to render 3D environment you need. These game engines involve 3D loaders which load the $3 \mathrm{D}$ content being used for the Virtual Tour and enable them to be manipulated using Flash ActionScript code.

Unity 3D / UE4 - As game engine these software frameworks support the creation of video games. Their main functionalities include a renderer for $2 \mathrm{D}$ and $3 \mathrm{D}$ graphics, collision detection, Sound, Scripting, AI, Localization and a scene graph.

Unity provides a platform to develop games for multiple platforms including IOS, Android, Windows, Mac, PS4, Xbox, Oculus rift and many more, supporting development for desktop, console, mobile, web and VR platforms. It also has real time global illumination and physically based shader. Unity combines both level editor, and programming languages including C\#, Boo and JavaScript to develop the application. As a professional tool, it supports a large number of images, sound and video formats to allow for easy importing.

UE4 is a game development tool that provides a platform to develop games for multiple platforms including IOS, Android, Windows, Mac, PS4, Xbox, Oculus rift and many more, supporting development for desktop, console, mobile, web and VR platforms. Unreal uses custom lighting, shading visual effects and cinematic systems. UE4 also combine a variety of tools and editors with programming using $\mathrm{C}++$.

Unreal also has a feature called blueprints, which allow you to develop game logic without using any code. It also has a terrain and foliage tool which allows you to create large open world environments, and then painting them with trees, grass and other terrains. It has a simple drag and drop system that allows the user to quickly and easily populate the virtual world. It is also Oculus rift compatible which is exactly what we need for the creation of this project.

To illustrate some features of these different digital content solutions, we propose a comparison of a series of virtual environment walkthrough applications developed over the years by LJMU students. The details can be shown in figures 1(a)-(h) and in table 1.

3D modelling and rendering techniques have improved drastically over the past few years, and we believe that a fully virtualized tour of an environment can offer a more immersive and interactive experience. Furthermore, it will be able to overcome the inherent limitations previously discussed. The remainder of this paper will build upon our idea of utilizing a fully virtualized and interactive tour environment, and we will compare (a) Scuderia Ferrari and (h) LJMU Formula Student Race Team in terms of four criteria: ease of navigation, level of interaction, visual appearance and information communication.

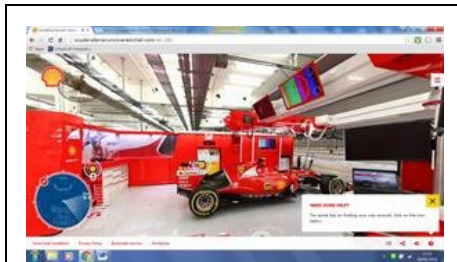

(a)- Scuderia Ferrari (WebGL, 2015)

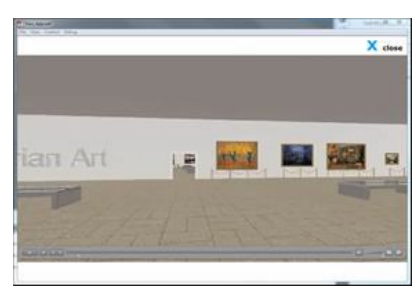

(c)-Museum VT (Away3D, 2012)

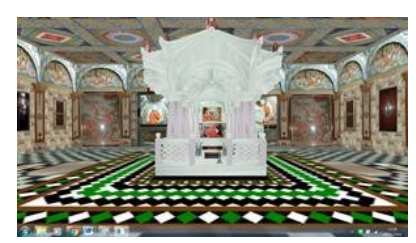

(e)-Deri Temple VT (OpenGL, 2007)

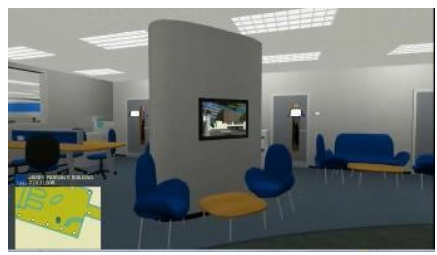

(g)-LJMU VT (Unity 3D, 2014)

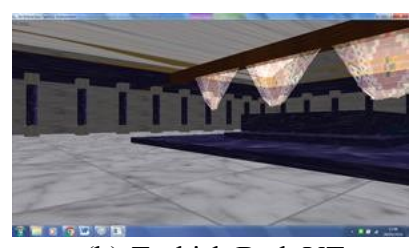

(b)-Turkish Bath VT (OpenGL,2006)

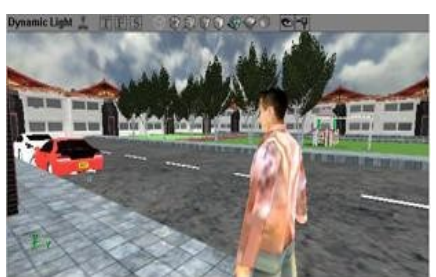

(d)-City VT (Homura 3D, 2011)

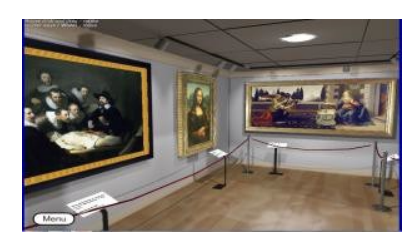

(f)-Museum VT (Flare3D, 2016)

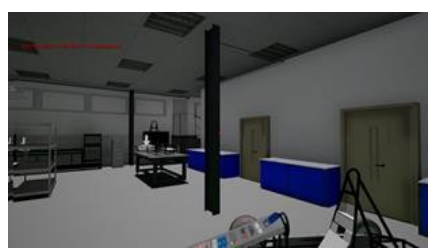

(h)- LJMU Formula Student Race Team (UE4, 2016)
Figure 1 (a)-(h). Virtual Tours

Table 1. Content Creation Tools Comparison

\begin{tabular}{|c|c|c|c|c|c|c|c|c|}
\hline vT & Platform & Lang & Web & Mobile & 30 & 20 & VR & $\begin{array}{c}\text { GPU } \\
\text { Support }\end{array}$ \\
\hline (a) & WebGL & is & Yes & Yes & N/A & $\begin{array}{l}\text { Photo- } \\
\text { Stitching }\end{array}$ & No & No \\
\hline (b) & Opengl & $\mathrm{CH}$ & & No & 305 & Texturing & No & No \\
\hline (c) & $\begin{array}{c}\text { Flash Away } \\
\text { 3D }\end{array}$ & A53 & Yes & Yes & $\begin{array}{l}\text {.DAE, } \\
\text {.FBX }\end{array}$ & Texturing & No & No \\
\hline (d) & Homura 3D & Java & Yes & No & .DAE & Texturing & No & No \\
\hline (e) & Opengl & $\mathrm{CH}$ & & No & 305 & Texturing & No & Yes \\
\hline (f) & $\begin{array}{c}\text { Hash } \\
\text { Hare3D }\end{array}$ & AS3 & Yes & Yes & DAE & Texturing & No & Yes \\
\hline (g) & Unity & $\begin{array}{c}\text { C\#, Boo, } \\
\text { is }\end{array}$ & Yes & Yes & . FBX & Texturing & Yes & Yes \\
\hline (h) & UEA & $\mathrm{CH+}$ & Yes & Yes & FBX & Texturing & Yes & Yes \\
\hline
\end{tabular}




\section{APPROACH}

The virtual tour developed in this paper focuses on the Formula Student Race Team project at Liverpool John Moores University. It is a self-sufficient organization that operates solely on endorsements and sponsorships from external companies. This could include either financial aids to fund the research and development costs or purchase parts, or even sending in equipment and parts for the vehicle and driver. The team also rely on the skills and talents of students to help their cause. They have dedicated staff working on different aspects of the vehicle, such as electrical engineers, engineers putting together the engine and welders building the chassis.

\section{A. Case Study}

Given the nature of the virtual tour, a dedicated case study was conducted prior to the development of the virtual tour. In this section of the paper, the focus of the research is on one related virtual tour project; the Scuderia Ferrari Garage tour.

\section{"It takes years of hard work to step foot into the Scuderia} Ferrari Garage. You can enter with one click."

Above is the opening quote to Scuderia Ferrari Uncovered; a $360^{\circ}$ degree Interactive Virtual pit-stop tour developed by Shell. The quote itself, sums up the benefits of virtual tours for information sharing. The Scuderia tool educates and informs users about the amount of labour and effort that goes into racing and maintaining the Scuderia Ferrari SF15-T

Formula 1 Race car during a race weekend. It carefully articulates itself to disseminate the most amount of information in a clean, minimal yet highly informative manner.

This Interactive Virtual application consists of a main hub, where the user can explore the Ferrari pit-stop and learn more about how it works. This is achieved by interacting with the environments hotspots located around the map, visualized on screen by yellow circles with pulsating rings around them. These represent interactive hotspots and are frequent throughout different areas of the tour. They are accessible by clicking different parts of the map such as: doorways or floor markings, using the google maps gizmo.
The system also allows users to explore the Ferrari pit-stop and learn more about its functionality. This is, again achieved by interacting with the environments through use of hotspots located around the 3D map. The UI's Map and Progress Meter monitor the user's progress within the virtual tour and output it to the screen so that the user can see where they are and how far into the tour, they are.

A drop down menu tab in the top left of the screen, enabling the user to see how many hotspots they have uncovered. This means that users are able to understand which ones have been viewed and those that have not. The built-in map allows the user to navigate around the virtual pit stop, jumping to different areas of the map. This is so that the user can see clearly identify which hotspots have been unseen, as well as where the user is located in the virtual environment.

Specifically, uncovered hotspots are red with white crosses in, while the ones yet to beviewed are yellow with black crosses inside. The use of colour gives the user a clear view of how much progress has been made in the tour environment. Overall the Scuderia virtual tour employs a variety of techniques which are applied to our implementation. In the following section, the approach for the system presented in this paper is put forward.

\section{B. Methodology}

The approach taken for the system is outlined in Figure 2. The system framework is divided into multiple modules which work together to provide interactive tours. The blueprints/input data involve any 3D environment construction request and all source material needed to produce the model. In the case of our research, this includes information about the scale, size and items within the student facility.

Blueprints are sent to the distributor which filters the data and provides construction information to the constructor module. The Constructor module divides the input between a texture and modelling process. Textures are created as 2D images with UVW maps.

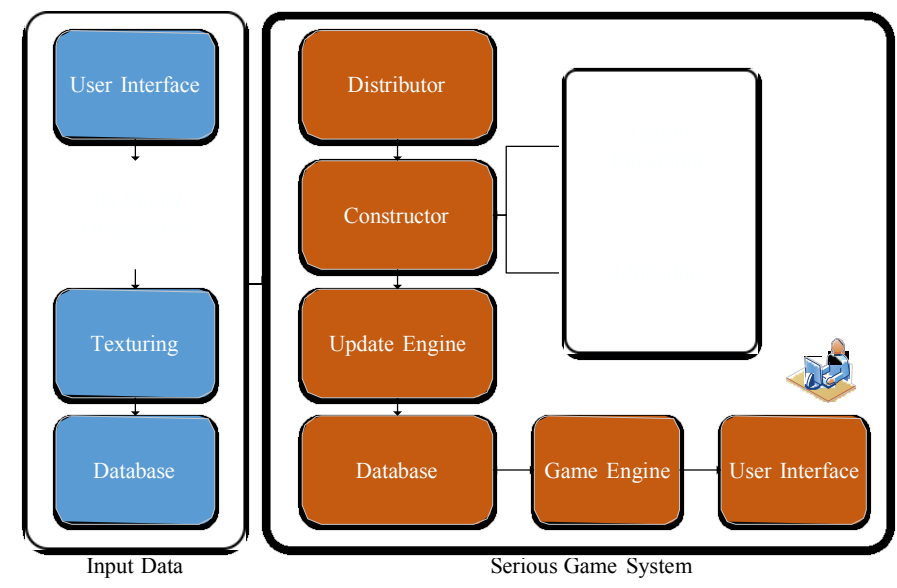

Figure 2. System Design 
The models are created in 3D. The Update Engine interacts between to the Constructor and Game Engine to update any content within the visualisation as needed. The future ambition of this project is to automate the process for the development of rapid virtual environment construction. However, for the purposes of the work presented in this paper, each of these stages was completed individually. The various development stages are presented in the following section.

\section{DEVELOPMENT}

The development process involved, first of all, understanding the game world being developed.

\section{A. The Game World}

The game world is a $3 \mathrm{D}$ environment built in Unreal Engine 4.10, consisting of 3D objects modelled by hand imported as static meshes. The objects are then scaled and placed accordingly, in order to create a highly accurate resemblance to the Formula Student Race Team Workshop Facility. As such, the process involves planning the following steps.

- World Layout: The layout is based heavily on the Formula Student Race Team Workshop, as such object position and locations, with constant on site visits to the workshop to ensure all objects and texture are presented and positioned correctly in the game world.

- Game Character: The player takes control of the game camera, which is essentially the player character, as the player role is simply that of a person playing the game with the intention of learning, therefore there's not much need for a player character besides a camera.

- Single Player Mode: In its current iteration, the LJMU Formula Student Interactive Virtual Tour consists of a single player game mode, where the player can explore and interact with a recreated environment, as evident in the gameplay that gives the user the ability to do these things.

The next stage involves texturing creations for the environment. The 2D texture creation is implemented using industry standard software. UVW maps are created and applied to the model in the Update Engine, as displayed in Figure 3.

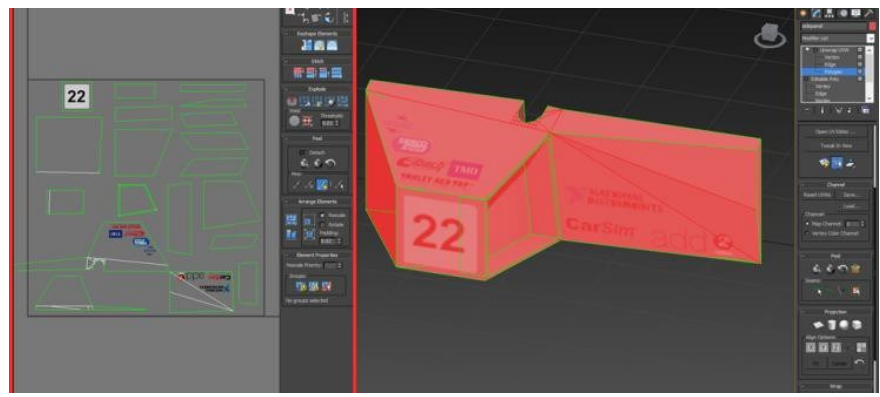

Figure 3. Texturing Process in UE4

\section{B. Modelling}

For the 3D asset creation, all files created are compatible with Unreal Engine 4.10. Firstly, the surrounding environment is developed; for example, the walls, ceiling and floor. However, the priority is creating a room that was to scale with the real environment, which requires up-to-date blueprints. The main focus is ensuring all objects created are both UV Mapped. This so that any textures applied appear correctly on the model and the UV coordinates is saved to the model upon exporting, and FBX Compatible, to be used in Unreal Engine 4. The 3D modelling process is conducted exclusively using quadrilateral polygons, as opposed to using triangular polygons. Using quadrilaterals is not a requirement to get a performant virtual tour; however, it is implemented in this research for the following reasons:

- Using quadrilateral polygons ensures that the polygon count is as low as possible without sacrificing quality. This enables for an accurate recreation of the environment without causing unnecessary frame rate lag.

- Quadrilateral polygons provide a more orderly topology for UV Mapping. Using triangular quads would require more focus on vertex manipulation. In other words, this ensures the texturing process is simplified.

\section{Game Engine}

Unreal Engine is selected for the construction as it is considered industry standard. Figure 4, presents a sample of the virtual world implementation process within the game engine.

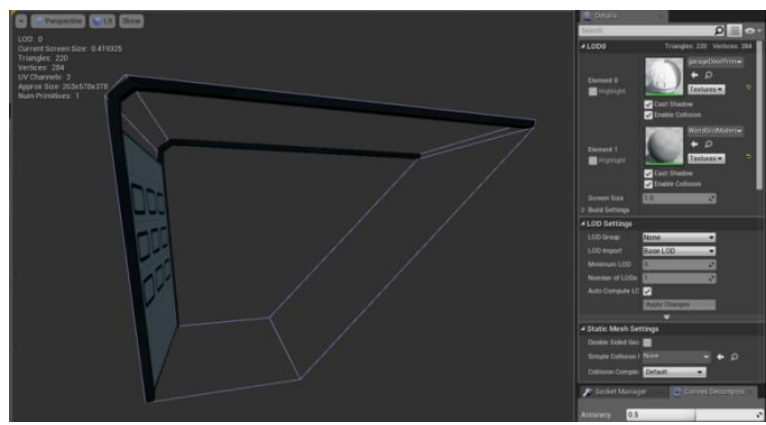

Figure 4. Collision Detection Process

It is a powerful game engine used by a wide range of companies in the gaming industry, from Android Indie Developers, to AAA Console Developers. Its use provides a wide range of tools to develop a fully functional and well-built artefact. It also possesses a rudimentary asset importer and editor to import models, textures, and sounds, and edit them extensively in the engine, and also with animation tools to script interactions and animations. The production pipeline involves importing the assets developed PNG and FBX files respectively. Collision volumes around key areas of the map are subsequently added in, in order to allow the user to interact with information screens. In Unreal Engine 4, when importing a 3D model, the engine automatically generates a 
convex collision map for the object that prevents the player, or certain object from passing through.

As shown in the Figure 4, the engine automatically inserts a collision box for the object, indicated by the blue lines around the object. However, in light of the shape of the object being concave, a custom collision box is required to be generated to better fit the object, and allow the user to pass underneath it.

The settings above allow the user to control how many vertices the collision box has, and also how accurate the collision map is to the $3 \mathrm{D}$ object, the settings above when applied, create a bounding box. Figures 5 to 8 present an overview of the interactive walkthrough tool and the main menu. The menu allows the user to change between screen sizes, and full screen mode, before returning to the main menu.

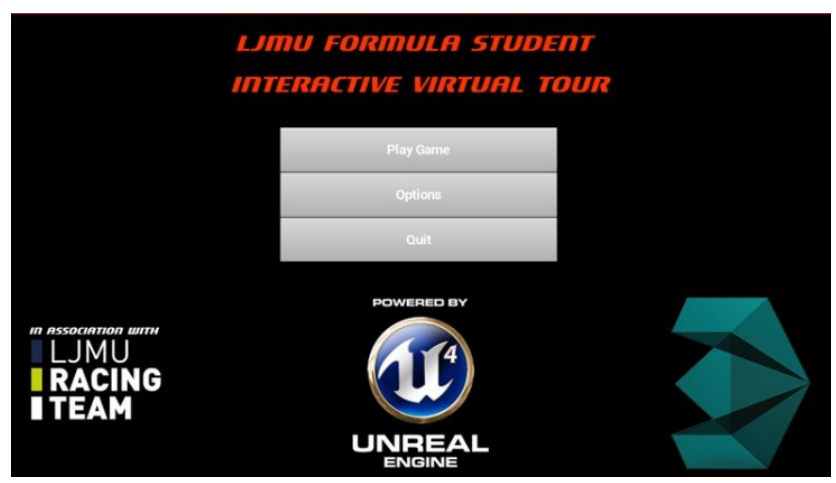

Figure 5. Welcome Menu

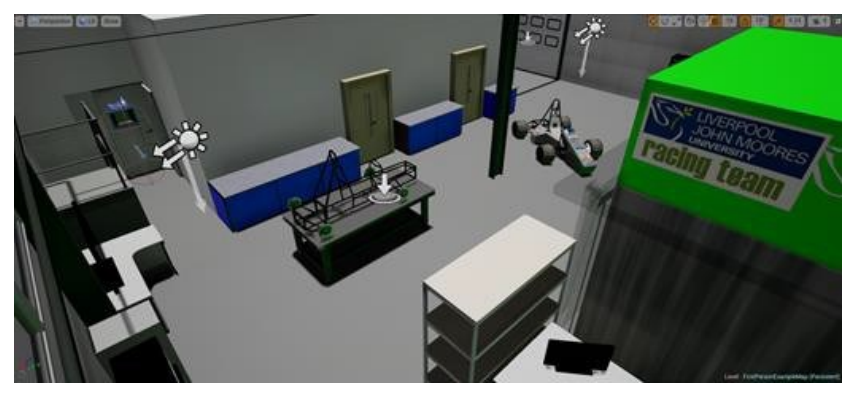

Figure 6. Environment View A

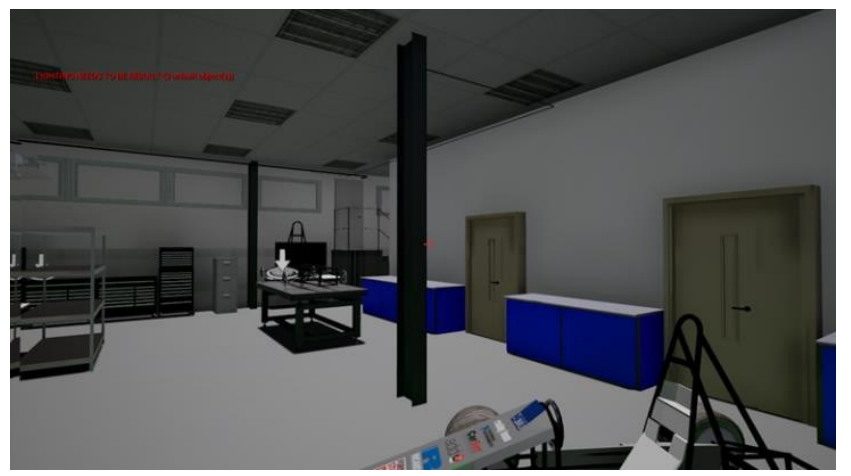

Figure 7. Environment View B

Figure 8 presents one of many information hotspot interaction setups; overall there is one per hotspots. Each hotspot is essentially a pause menu, and acts like, but just references a different screen.

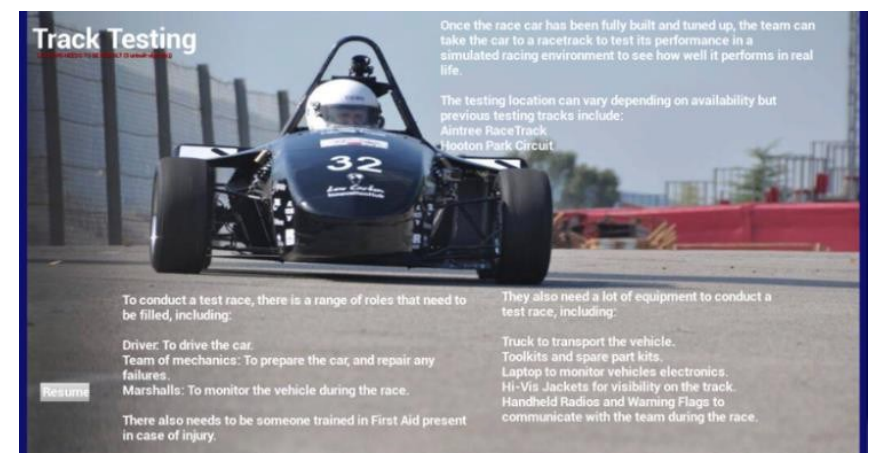

Figure 8. Information Point A

The user interacts with the system through the keyboard, and specifically, when the ' $\mathrm{E}$ ' key is pressed, the information can be accessed. These functions by use of a gate that checks if Button press is TRUE or FALSE and checking to see if the player is overlapping with the trigger volume in the world. If both are TRUE then it checks to see if the input is valid, and creates the information hotspot widget, and creates a reference, which is then linked to a validity check to confirm that the action can be completed.

The player controller, gets a reference to the widget, and sets the input mode to UI only to remove any controls that could conflict with the menu. This then adds the created widget to the screen and pauses the game. Following on from the implementation, the virtual tour is evaluated in the following section.

\section{EVALUATion}

When the user selects to play the game, he is taken to the main environment they are able to explore the student facility environment. When selected, information hotspots open up a window that provides data about the object. In order to evaluate the finished artefact, a user testing process was conducted with a group of students aged $18-24$. The students completed questionnaires using the Likert scale from 1 to 5 to assess the effectiveness. Specifically, the tool was assessed on four criteria: ease of navigation, level of interaction, visual appearance and information communication.

\section{A. Qualitative Assessment}

Each user study lasted 15 minutes including briefing on the interaction controls. The results are presented in Table 2 below.

Table 2. User Evaluation

\begin{tabular}{ll}
\hline Assessment & Score (mean \%) \\
\hline Ease of navigation & 4.75 \\
Level of interaction & 3.75 \\
Visual Appearance & 4.13 \\
Information Comm. & 3.63 \\
\hline
\end{tabular}


In addition to the above, the students were asked to compare the artefact to a control model; specifically, the Scuderia Ferrari Uncovered (SFU) presented in Section 3. An artefact preference metric was added to each section of the questionnaire presented above. Students were asked to select their preference for each tool based on the fours assessment criteria. This was a simple qualitative question, where the students could only select one or the other. The results are presented in Table 3 as percentage scores of the user choice.

Table 3. Artefact Comparison

\begin{tabular}{lll}
\hline Assessment & LJMU & SFU \\
\hline Ease of navigation & $75 \%$ & $25 \%$ \\
Level of interaction & $75 \%$ & $25 \%$ \\
Visual Appearance & $75 \%$ & $25 \%$ \\
Information Comm. & $50 \%$ & $50 \%$ \\
\hline
\end{tabular}

The overall mean score for the user evaluation process was $4.065(81.3 \%)$. In addition, a mean average of $68.75 \%$ of users preferred the tool created to case study comparison for each assessment. As the SFU was developed by a professional team of developers, the above results are unexpected. However, SFU virtual tour is developed by Spherical Photo Stitching techniques. This allows for the creation of highly detailed maps for the user to explore, along with responsive functionality. However, Photo Stitching methods depend upon the suitably of captured photos, which in turn restricts the approach to photographed places and objects. The Photo Stitching approach makes use of pre- positioned points for exploring the environment.

Whereas, the LJMU tool was developed as a 3D graphicalbased approach, which means the content can be updated dynamically and the interaction possibilities are higher. This was reflected in the results, where $75 \%$ of users selected the LJMU tool as having a higher level of interaction.

\section{B. KLM Analysis Comparison}

To complete the assessment, a KLM analysis is conducted on four tasks: Opening Artefact from main menu, activating a close hotspot, activating a faraway hotspot and accessing the map. The results of both tools are presented in Table 4 below. The 3D walkthrough compared with a Photo Stitching approach is displayed. All timings displayed are based on industry standard operation times for the corresponding action. However, slight modifications have been included to incorporate holding keys down for movement in the environment.

Table 4. KLM Analysis.

\begin{tabular}{|c|c|c|c|c|c|c|c|}
\hline Scuderia Ferrari Uncovered & & & & LJMU Formula Student Virtual Tour & & & \\
\hline Opening Artefact from main menu & & & & Opening Artefact from main menu & & & \\
\hline Mentally Prepare for action & M & 1.35 & & Mentally Prepare for action & M & 1.35 & \\
\hline Point mouse at target & $\mathrm{P}$ & 1.1 & & Point mouse at target & $\mathrm{P}$ & 1.1 & \\
\hline Left click & $\mathrm{K}$ & 0.1 & & Left click & $\mathrm{K}$ & 0.1 & \\
\hline Total time & & 2.55 & Seconds & Total time & & 2.55 & Seconds \\
\hline Activating to a close hotspot & & & & Activating to a close hotspot & & & \\
\hline Mentally Prepare for action & M & 1.35 & & Mentally Prepare for action & M & 1.35 & \\
\hline Point mouse at target & $\mathrm{P}$ & 1.1 & & Hold down 'W Key' to move forward (1sec) & $\mathrm{k}^{* 1}$ & 0.06 & \\
\hline Left click & $\mathrm{K}$ & 0.1 & & Point Mouse at target & $\mathrm{P}$ & 1.1 & \\
\hline Total time & & 2.55 & Seconds & Key Press & $\mathrm{K}$ & 0.1 & \\
\hline & & & & Total time & & 2.61 & Seconds \\
\hline Activating a far hotspot & & & & Activating a far hotspot & & & \\
\hline Mentally Prepare for action & M & 1.35 & & Mentally Prepare for action & M & 1.35 & \\
\hline Left click & $\mathrm{K}$ & 0.1 & & Point mouse at target & $\mathrm{P}$ & 0.33 & \\
\hline Point mouse at target & $\mathrm{P}$ & 1.1 & & Hold key to walk towards target & $\mathrm{K} * 2$ & 0.12 & \\
\hline Left click & $\mathrm{K}$ & 0.1 & & Key Press & $\mathrm{K}$ & 0.1 & \\
\hline Point mouse at target & $\mathrm{P}$ & 1.1 & & Total time & & 1.9 & Seconds \\
\hline Left click & $\mathrm{K}$ & 0.1 & & & & & \\
\hline Total time & & 3.85 & Seconds & & & & \\
\hline Access map & & & & Access map & & & \\
\hline Mentally Prepare for action & M & 1.35 & & Mentally Prepare for action & M & 1.35 & \\
\hline Point mouse at target & $\mathrm{P}$ & 1.1 & & Key Press & $\mathrm{K}$ & 0.1 & \\
\hline Left click & $\mathrm{K}$ & 0.1 & & Total time & & 1.45 & Seconds \\
\hline Total time & & 2.55 & Seconds & & & & \\
\hline
\end{tabular}


Table 5. KLM Analysis.

\begin{tabular}{lclc}
\hline SFU Total Operation Time & $\mathbf{1 1 . 5}$ & 3D Tool Total Operation Time & $\mathbf{8 . 5 1}$ \\
\hline Average task time & 2.875 & Average task time & 2.1275 \\
\hline
\end{tabular}

Table 5 displays the overall mean average task time for the SFU Photo Sketch environment compared to the LJMU 3D walkthrough. Using Table 4 as a reference point, it is possible to calculate and compare individual tasks. For example, accessing activating a close hotspot, the calculations are as follows:

$$
\begin{gathered}
S F U=M+P+K \\
L J M U=M+(K * n)+K
\end{gathered}
$$

Scuderia Ferrari Uncovered is more efficient by 0.06 seconds. This due to a modified calculation within LJMU Virtual Tour which calculates key press by the time it is held down. Within the SFU environment, hotspots are activated by clicking them on the screen; whereas LJMU Virtual Tour requires the user to ' walk' up to them and interact.

Activating a far hotspot is calculated as follows:

$$
\begin{gathered}
S F U=M+K+P+K+P+K \\
L J M U=M+\left(P^{*} n\right)+\left(K^{*} n\right)+K
\end{gathered}
$$

In this case, the LJMU 3D walkthrough is more efficient by a 1.95 margin. This is due to users having to click and drag the screen multiple times in Scuderia to be able to interact with a hotspot. Within the 3D modelled environment, the user only has to walk over for an interaction. Overall, the total task times in seconds for the artefacts were: 11.5 for Scuderia, and 8.51 for LJMU, which average times being 2.875 and 2.1275 respectively.

\section{CONCLUSION AND FUTURE WORK}

Specific considerations for the advancement of this research include the following:

- Console Support: One fact I discovered through testing, was that a majority of users had access to a gaming console, as well as a PC, the issue with $\mathrm{PC}$ is that each PC is different hardware wise, and has differing responses to my application, whereas a console version would work on any console, and can reach more people effectively, so this is an area of interest.

- Online Multiplayer: As the student facility is a team project, the virtual tour is intended for use at open days and fresher fairs. Therefore, the first consideration is that it will be incorporated into an online mode for users to group up and explore, with assigned moderators in-game to help inform and enhance the experience of the user.

- Racing Sim: Due to the nature of the team, and the development made on the simulator by researchers, it is possible to create a $3 \mathrm{D}$ simulation based on the actual car, which could be imported into Unreal Engine, and create a racing mini-game to demonstrate driver training.

- Controller and VR support: Both these aspects are considered for a future implementation process. The use of VR will allow users to look around in depth, taking in more detail, and the controller will provide a more ergonomic method of control for the users.

Acknowledgments: The last author's research work is supported by national projects with grant no: 61332017 and 2015BAK04B00.

\section{REFERENCES}

[1] S. Okajima., \& Y. Okada, Treecube 3D-ViSOM: Combinational Visualization Tool for Browsing 3D Multimedia Data, 11th International Conference on Information Visualization, IV '07, pp 4045,2007

[2] H. Xu., W. Yu., L. Wei., N. Zhang., et al., An interactive system for heterogeneous 3D volumetric data visualization, 5th International Conference on Computer Science and Education (ICCSE), pp 24-27, 2010

[3] D. Walsh, World Heritage in Google Earth: 3D Models Across the Globe, Available at [http://www.cyark.org/news/world-heritage-ingoogle-earth-3d-models-across-the-globe], Accessed, June 2016

[4] Arttube, Videos About Art and Design, Available at [http://www.arttube.nl/en?gclid=CKG3hufqve0CFcsp0wodCGgMxQ], Accessed June 2016

[5] W. Woodard., \& S. Sukittanon., Interactive virtual building walkthrough using Oculus Rift and Microsoft Kinect, SoutheastCon, pp 1-3, 2015

[6] L. Avila., \& M. Bailey., Augment Your Reality, IEEE Computer Graphics and Applications (Volume:36, Issue: 1), pp 6-7, 2016.

[7] Bonacini, E., The Paolo Orsi Syracuse Archaeological Museum pilot project, in 2015 11th International Conference on Signal-image technology \& Internet-based Systems, Bangkok, Thailand, 2015

[8] Fineschi, A. \& Pozzebon, A., A 3D Virtual Tour of the Santa Maria Della Scala Museum Complex in Siena, Italy, based on the use of Oculus Rift HMD, in 2015 International Conference on 3D Imaging (IC3D), Liege, 2015

[9] Gonzalez-Delgado, J. A., Martinez-Grana, A. M., Civis, J., Sierro, F. J., Goy, J. L., Dabrio, C. J., Ruiz, F., Gonzalez-Regalado, M/ 1/ \& Abad, M., Virtual 3D tour of the Neogene paleontological heritage of Huelva (Guadalquivir Basin, Spain), Environ Earth Sci, vol. 73, no. 8, pp. 4609-4618, 2014

[10] Cosido, O., Teran, L., Lopez, O. R., Sarachaga, M., Campi, M., Catuogno, R., Gutierrez, N. G. \& Latorre, E. M., 3D Tour through University Spaces for the Management and Dissemination of the Cultural Heritage of the University of Cantabria, in 2015 Digital Heritage, Granada, 2015.

[11] Noordin, M., Ismail, I. \& Yahya, M., Virtual Tourism Application through 3D Walkthrough: Flor De La Mar, in 7th International Conference on Information Technology (ICIT), Seville, 2015.

[12] Zhang, D., Zheng, R. \& Yang, G., Image Clusters Based 3D Virtual Tour Schema, in 5th International Conference on Software Engineering and Service Science (ICSESS), Beijing, 2014.

[13] Alletto, S., Abati, D., Serra, G. \& Cucchiara, R., Exploring Architectural Details Through a Wearable Egocentric Vision Device, Sensors, vol. 16 , no. 2, 2016

[14] Chen, F, \& Mo, H., Users' Perspectives on and Learning Effectiveness of Tour-Guide Training Courses With 3D Tourist Sites, Journal of Teaching in Travel \& Tourism, vol. 13, no. 4, pp. 374-390, 2013.

[15] C. Silenzi., Software Engineering in Ferrari F1, 37th IEEE International Conference on Software Engineering, vol. 1, pp 1, 2015 